\title{
Application de l'hologiraphie ultra-rapide à la détermination de la taille et de la vitesse des particules dans un écoulement diphasé
}

\author{
par A. Kleilz \\ Electricité de France, \\ Direction des Etudes \\ et Recherches
}

L'étude des broulllards, des aérosols, des pulvérisations de liquide, etc., nécessite la détermination de la taille et de la vitesse de particules très fines, liquides ou solides, animées de vitesse plus ou moins importante. Les procédés classiques ont des inconvénients : perturbation du milieu par la présence de l'appareillage (dans les techniques des empreintes), profondeur de champ très réduite (en microphotographie), obtention de mesures indirectes ou globales etc.

\section{Pourquoi l'holographie}

On sait que l'holographie est une méthode optique qui permet de reconstituer, en trois dimensions, l'image d'un objet à partir d'un seul enregistrement. Les récents progrès des lasers à rubis, et plus particulièrement la mise au point du laser déclenché monomode (impulsion de très courte durée de 10 à $30 \cdot 10^{-9} \mathrm{~s}$ ), permettent maintenant d'holographier des objets en mouvement : c'est l'holographie ultra-rapide. Dans le cas de particules, on obtient les images de celles-ci comme "figées " dans l'espace; il est alors possible de les observer, de les mesurer à loisir. L'holographie ultra-rapide est donc apparue comme particulièrement attrayante dans ce domaine, car elle fournit une mesure directe sans perturber l'écoulement.

Dans ce qui suit, nous exposerons le principe du montage particulier utilisé, sans développer la théorie et les résultats généraux de l'holographie, en renvoyant pour cela les lecteurs aux nombreux ouvrages spécialisés signalés dans les références bibliographiques, puis nous donnerons quelques indications sur les limites pratiques de la méthode, déduites en particulier des essais que nous avons effectués.

\section{Principe du montage et mode opératoire}

Dans le cas des particules, des gouttelettes d'eau par exemple, en suspension dans un milieu homogène (air, vapeur), on utilise le montage de "Gabor " en lumière parallèle illustré par les figures 1 et 2. Les opérations s'effectuent en deux temps :

\section{L'enregistrement (fig. 1)}

Un faisceau de lumière parallèle, issu du laser pulsé, vient éclairer une plaque sensible $H$ après avoir traversé le brouillard à étudier. On enregistre les interférences entre la lumière diffractée par chaque particule et la lumière directement transmise qui constitue l'onde de référence. Le système de franges qui en résulte constitue l'hologramme (cliché $n^{\circ}$ 6).

La restitution (fig. 2)

Un faisceau de lumière parallèle, issu d'un laser continu (onde de restitution), éclaire l'hologramme précédemment obtenu; celui-ci se comporte comme un réseau de diffraction et la théorie de l'holographie montre qu'il y a au moins alors formation de deux images, l'une virtuelle (image directe), l'autre réelle (image conjuguée).

Effectuons une étude simplifiée sur une particule sup. posée ponctuelle. Soit $a_{0}$ et $F$ respectivement l'amplitude complexe due à l'onde de référence et celle de l'onde 
issue de la particule. La plaque sensible a reçu, à l'enregistrement, une amplitude $a+F$ et un éclairement égal au carré de cette amplitude oomplexe soit :

$$
E=\left(a_{0}+F\right)\left(a_{0}^{*}+F^{*}\right)
$$

$F^{*}$ étant l'onde conjuguée de $F$ (pour l'onde de référence plane $a_{0} *=a_{0}$. Après développement, le négatif obtenu (hologramme) donne par transmission une amplitude propontionnelle à $E$. On montre que cette dernière peut se mettre sous la forme :

$$
b=b_{0}-\alpha\left[F^{2}+a_{0} F+a_{0} F^{*}\right]
$$

Les deux premiers termes $\left(b_{0}-\alpha F^{2}\right)$ représentent pratiquement l'onde directement transmise. Le terme $-\alpha a_{0} F$ représente l'onde qui reconstitue une image virtuelle en $G$ ', et au terme $-\alpha a_{0} F^{*}$ correspond l'onde qui reconstitue une image réelle en $G^{\prime \prime}$,

\section{Dépouillement}

Si les longueurs d'onde des deux lasers étaient égales, $G^{\prime}$ occuperait par rapport à l'hologramme la même position que la particule par rappont à la plaque sensible vierge, et $G^{\prime \prime}$ une position symétrique. Les longueurs d'onde sont légèrement différentes et on $\mathrm{a}$, en appelant $\lambda_{1}$ la longueur d'onde du laser pulsé et $\lambda_{2}$ celle du laser continu :

$$
Z^{\prime}=Z \lambda_{1} / \lambda_{2} \quad \text { et } \quad Z^{\prime \prime}=Z \lambda_{2} / \lambda_{1}
$$

Par contre, le grandissement pour toutes les images est constant et égal à l'unité.

L'image virtuelle est observable à l'œil nu. C'est surtout sur l'image réelle que l'on effectue les mesures de taille, soit par observation au microscope, soit par l'intermédiaire d'un circuit de télévision. Dans ce dernier procédé, on projette sur la face d'entrée du tube d'une caméra de télévision une image agrandie $G^{\prime \prime}$, donnée par un système optique simple, de l'image restituée $G^{\prime \prime}$; cette particule apparaît alors nette sur l'écran du récepteur, les particules en dehors du champ sont entourées d'une sorte de halo (fig. 3). Connaissant le grandissement de l'ensemble il est alors aisé de déduire la dimension réelle de la particule.

En déplaçant l'hologramme par rapport à la caméra, ou inversement, on explore tout le volume initial par des mises au point successives sur chaque particule restituée.

La visualisation en surimpression sur l'écran du récepteur d'une grille étalon facilite le dépouillement.

\section{Mesure de la vitesse par double exposition}

Sur le laser à rubis, un interrupteur optique à cellule de Pockels permet de déclencher deux impulsions rapprochées ; l'écart est ajustable de 2,5 à $50 \mu$ s. La restitution des images d'une particule holographiée à deux instants différents isur une même plaque donne la vitesse en grandeur et en direction.

\section{Avantages de la méthode holographique}

Les avantages de l'holographie et en particulier de ce montage sont nombreux :

- restitution des particules dans l'espace, par des images virtuelles et réelles ;

- mesure directe de leur taille et de leur vitesse;

- simplicité, tant pour la prise de l'hologramme que pour la restitution; en particulier, les problèmes d'alignement optique et de réglage de l'intensité ne sont pas critiques ;

- haute résolution : limite inférieure de l'ordre de cinq miorons :

- pas d'abenrations géométriques pour les images;

- possibilité d'analyser des volumes importants; quelle que soit la position de la goutte par rapport à la plaque, la différence de chemin optique entre les rayons lumineux diffractés par la goutte et ceux constituant le fond cohérent reste faible;

- possibilités d'enregistrer des gouttes animées de vitesses relativement élevées. Des essais ont été effectués jusqu'à des vitesses de $50 \mathrm{~m} / \mathrm{s}$.

\section{Domaine d'application et limites de résolution}

Différents facteurs influent sur la qualité des images restituées. Les plus critiques et ceux qui limitent la résolution de l'hologramme sont les suivants :

\section{Condition d'éloignement}

Soit $d$ le diamètre de la particule supposée sphérique. Il existe une distance particule-plaque minimale à respecter; cette distance peut être définie par $z_{1}=N_{1} d^{2} / \lambda$, avec $N_{1}$ variant de 1 pour les petites particules $(10$ à $100 \mu)$ à 0,1 pour les gouttes plus grosses (1 à $2 \mathrm{~mm}$ ).

Ex. :

$$
\begin{array}{ll}
d=10 \mu & z_{1} \sim 0,1 \mathrm{~mm} \\
d=100 \mu & z_{1} \sim 10 \mathrm{~mm}
\end{array}
$$

\section{Bruit de fond}

Si $z$ est supérieur de 50 à $100 d^{2} / \lambda$, l'information provenant de la panticule est noyée dans le bruit de fond, dû au grain de l'émulsion en particulier.

Ex. : valeur limite pour $z_{2}$ :

$$
\begin{array}{ll}
d=10 \mu & z_{2} \sim 1 \mathrm{~cm} \\
d=100 \mu & z_{2} \sim 1 \mathrm{~m}
\end{array}
$$

C'est ce qui se produit lonsqu'on est obligé d'éloigner la plaque sensible de l'écoulement. On peut y remédier en utilisant un système de transport d'image qui reporte l'objet dans le voisinage de l'hologramme (fig. 4) 


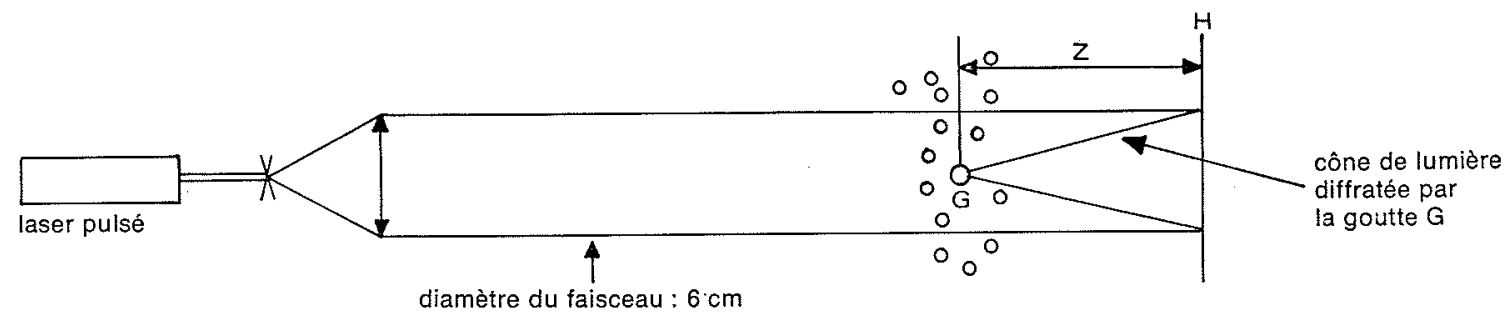

$1 /$

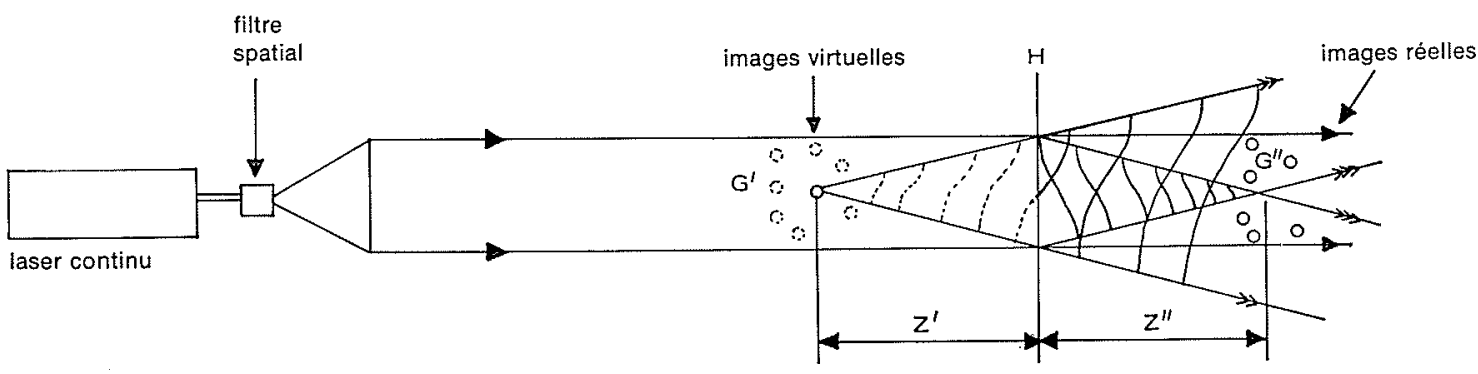

2/

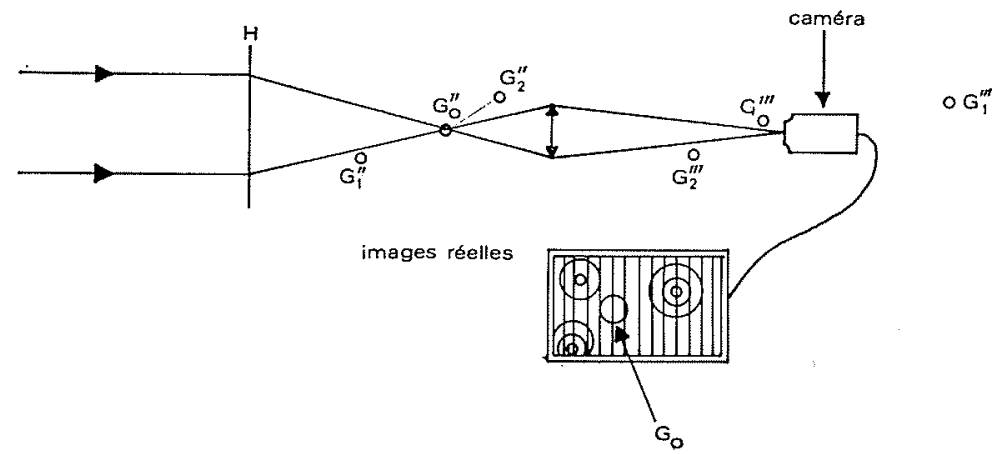

3/

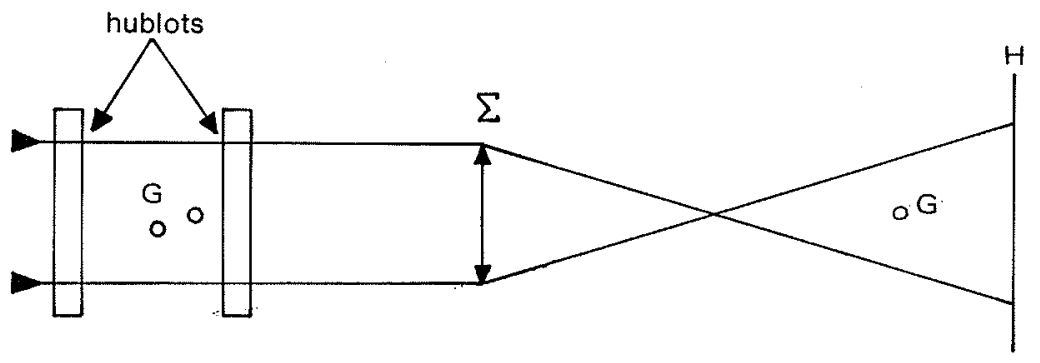

4/ 


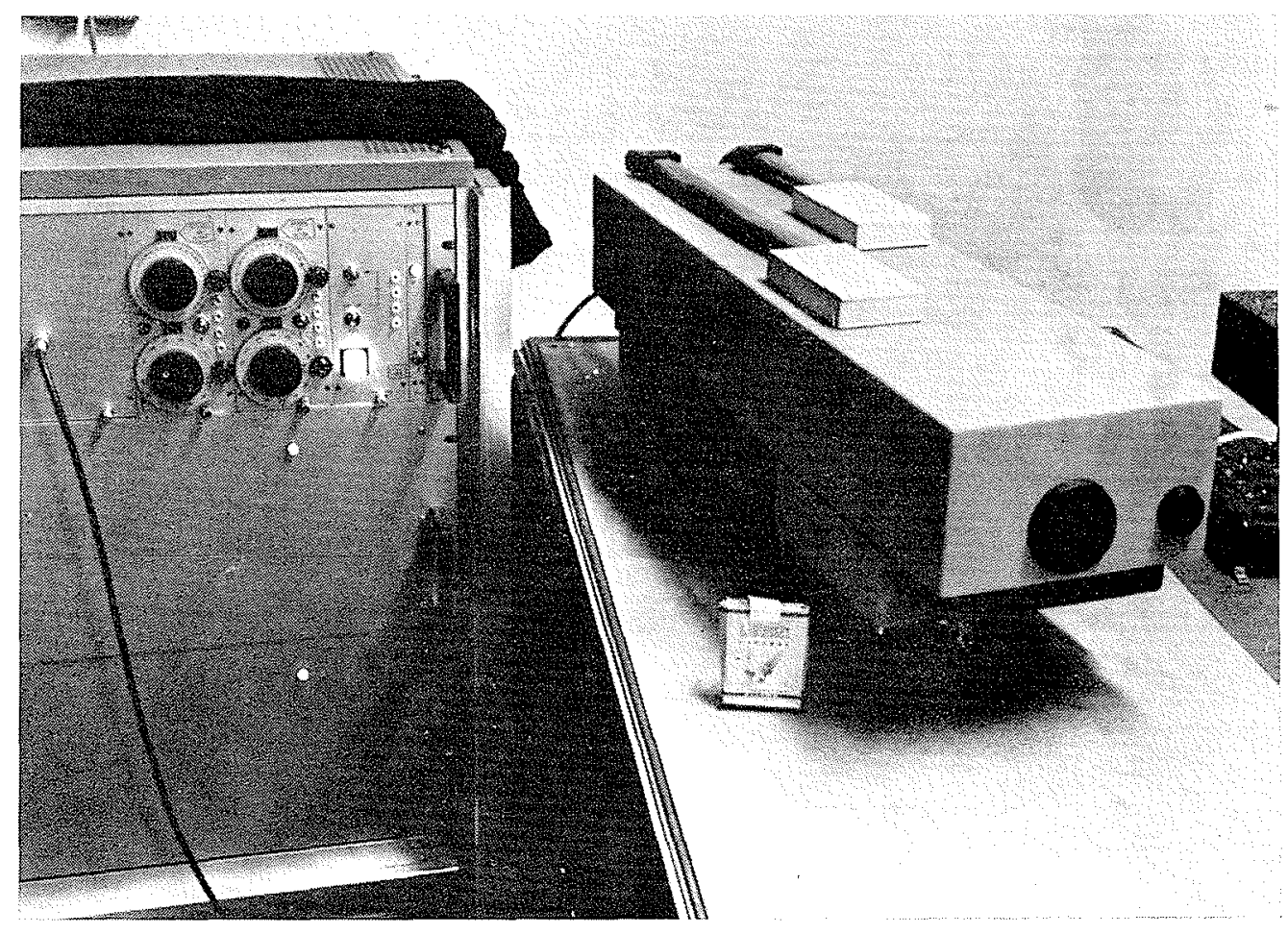

LASER PULSÉ UTILISÉ POUR L'ENREGISTREMENT DES HOLOGRAMMES

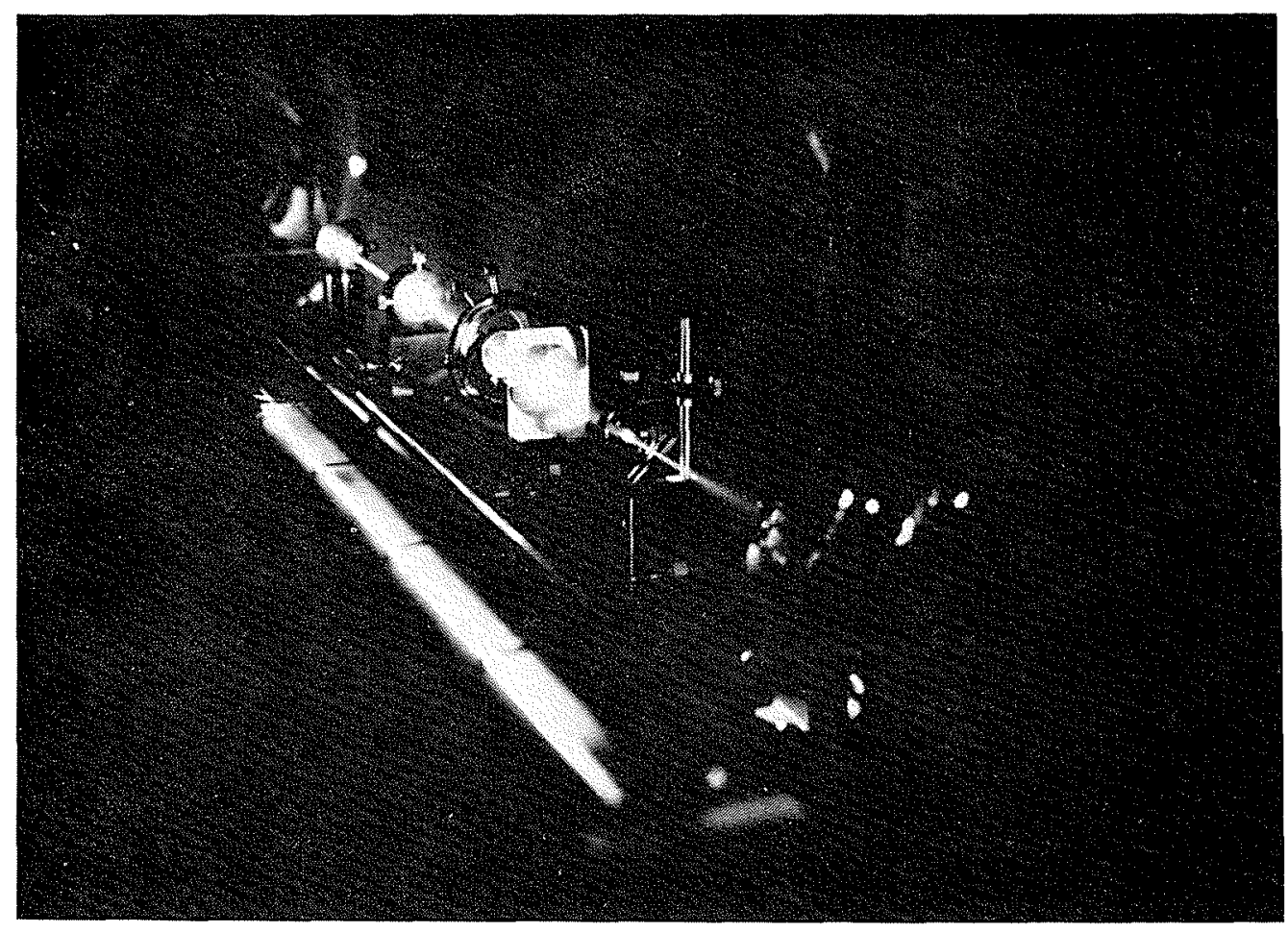

MONTAGE DE RESTYTUTION 
$G$, image $\mathrm{G}_{0}$ à travers $\Sigma$, joue le rôle d'objet pour l'hologramme $\mathrm{H}$; le faisceau de référence est alors divergent.

Si la restitution s'effectue en faisceau parallèle, le grandissement transversal est indépendant des longueurs d'onde et de la position de l'objet par rapport au système optique.

\section{Densité du brouillard}

La quantité d'informations que l'on peut stocker dans l'hologramme est naturellement limitée. Un trop grand nombre de particules conduit à l'enregistrement, dans l'émulsion sensible, d'une multitude de réseaux de franges enchevêtrées; il y a alors destruction plus ou moins totale de chaque réseau. D'autre part, le fond cohérent est détruit. Le nombre maximum $\mathfrak{x}$ de particules que l'on peut restituer par unité de surface d'hologramme est de l'ordre de $(d / \lambda z)^{2}$ et la concentration limite $c$ de l'ordre $\mathscr{K} / \Delta z, d$ étant le diamètre moyen des particules, $z$ la distance moyenne de celles-ci à la plaque, $\Delta z$ l'épaisseur du «brouillard".

\begin{tabular}{|r|c|c|r|r|r|}
\hline$d$ & $z$ & $\log (d / \lambda z)^{2}$ & $\begin{array}{r}\log \mathscr{Y} \\
\text { évalué } \\
(\mathscr{T} \\
\left.\text { en } \mathrm{cm}^{-2}\right)\end{array}$ & $\begin{array}{c}\Delta z \\
(\mathrm{~cm})\end{array}$ & $\begin{array}{c}c \\
\left(\mathrm{~g} / \mathrm{cm}^{3}\right)\end{array}$ \\
\hline & $(\mathrm{cm})$ & & 2,3 & 0,5 & 1800 \\
20 & 1 & 2,6 & 1,5 & 0,5 & 100 \\
20 & 4 & 1,7 & 2,2 & 5 & 40 \\
100 & 10 & 2,3 & 1,7 & 5 & 25 \\
100 & 20 & 1,7 & & \\
\hline
\end{tabular}

Pour les applications pratiques, c'est ce oritère qui limite le plus souvent l'emploi de la méthode.

\section{Qualités des optiques}

Lorsqu'il est nécessaire de prévoir des hublots (milieu en pression ou dépression, etc.), ou dans les montages avec repori d'objet, les optiques employées doivent être de très bonne qualité (qualité interférométrique) sinon il y aurait destruction de l'onde de référence. D'autre part, il faut éviter tout dépôt de particules ou toute condensation de buées.

\section{Relative lenteur du dépouillement}

Le développement de la plaque puis le dépouillement de l'hologramme (observation particule par particule) peut constituer un certain handicap.

\section{Matériels}

Laser pulsé (pour l'enregistrement de l'hologramme)

La réalisation d'un hologramme exige une source de lumière cohérente (c'est-à-dire suffisamment ponctuelle et monochromatique).

On a utilisé un laser à rubis monomode déclenché (modèle HR 17 de la Société Quantel) dont les caractéristiques sont les suivantes:
- Energie par impulsion ....... $20 \mathrm{~mJ}$

- Durée de l'impulsion ........ 10 à 30 ns $\left(10^{-9} s\right)$

- Longueur de cohérence ....... $\sim 1,50 \mathrm{~m}$

— Longueur d'onde ............ $\lambda=6943 \AA$

- Diamètre du faisceau ........ $\sim 3 \mathrm{~mm}$

- Divergence .............. 310.4 radian

La très courte durée de l'impulsion permet d'holographier des objets en mouvement et d'autre part de s'affranchir des probièmes de vibrations des supports (il n'est pas nécessaire d'utiliser des bancs d'optique spécialisés).

\section{Laser continu}

Pour la restitution, on a utilisé un laser continu héliumnéon (modèle $125 \mathrm{~A}$ de la Société Spectro-Physios) d'une puissance de $70 \mathrm{~mW}$ et d'une longueur d'onde $\lambda=6328 \AA$.

\section{Plaques sensibles}

Elles doivent avoir une très haute résolution $\left(2000 \mathrm{~mm}^{-1}\right)$, car les franges d'interférences à photographier sont très fines; on a utilisé des émulsions "Scientia 10 E 75 » d'Agfa-Gevaent (sensibilité $20 \mathrm{erg} / \mathrm{cm}^{2}$ ).

\section{Résultats et exemples d'applications}

Les clichés présentés dans les planches qui suivent sont des photographies des images restituées (réelles et virtuelles) obtenues à l'aide d'une loupe binoculaire. La méthode a été testée pour différentes gammos de diamètre de gouttelettes au cours de ces applications.

\section{Conclusion}

Dans les limites d'application que nous avons tenté de définir précédemment, nous considérons cette méthode comme opérationnelle. L'analyse direote des particules dans leur milieu d'origine permet des mesures de granulométrie non accessibles par les voies classiques, elle peut aussi fournir un étalonnage pour d'autres moyens de mesure. D'autre part la mise en cuvre des opérations, tant pour l'enregistrement que pour les phases de restitution ot de dépouillement, est relativement aisée; les matériels - laser pulsé et laser continu — sont actuellement fiables et d'emploi immédiat.

\section{Rétérences}

[1] Upatnieks (J.), Trolinger (J. D.), Menzel (R.), Farmer (M.), MATTHEWS (B. J.), WUerker (R. F.). - «Laser Technology in aerodynamic measurements ». Institut von Karman, Bruxelles (juin 1971).

[2] FRANCON. - «Holographie». Masson (1969).

[3] Marquet (M.). - Performances en Holographie. Revue d'Optique (septembre 1966).

[4] THOMPSON (B. J.), ZlNKY (W. R.). - Holography detection of submicron particles. Appl. Optics (1968).

[5] Fourney (M. E.), Matkin (J.H.), Whaoner (A. P.). - Aerosul size and velocity determination holography. Rev. Sci. Inst. (1969). 


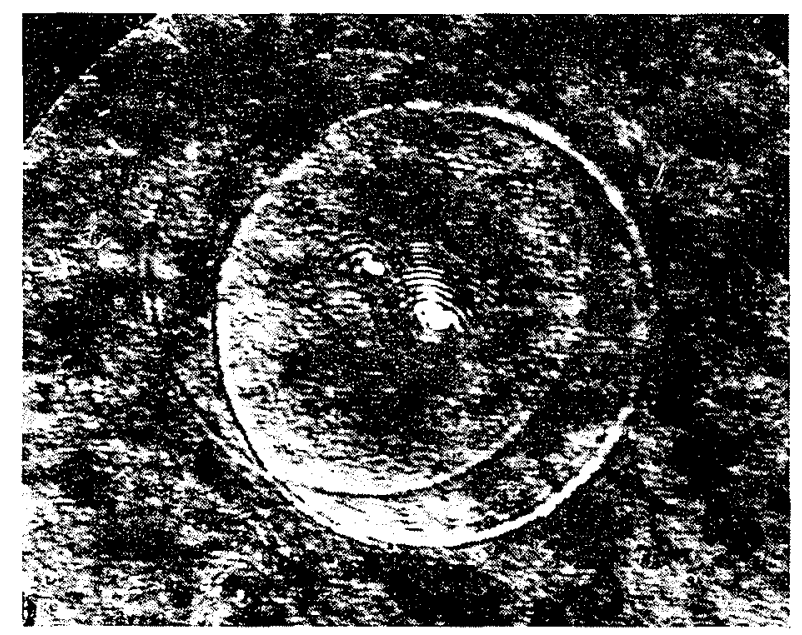

$$
d \sim 900 \mu
$$$$
N=0,6
$$

grandissement $g=50$

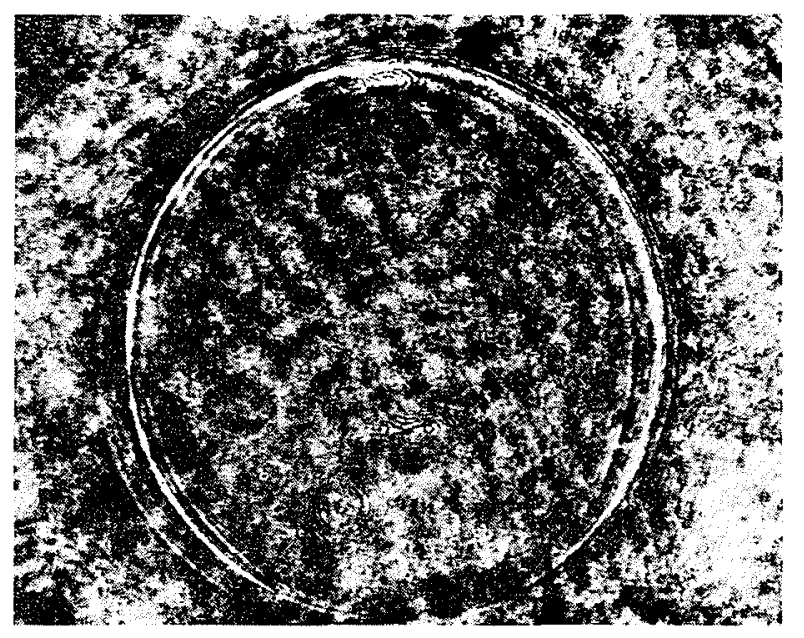

$$
\begin{aligned}
d & \sim 1800 \mu \\
N & =0,1 \\
g & =30
\end{aligned}
$$

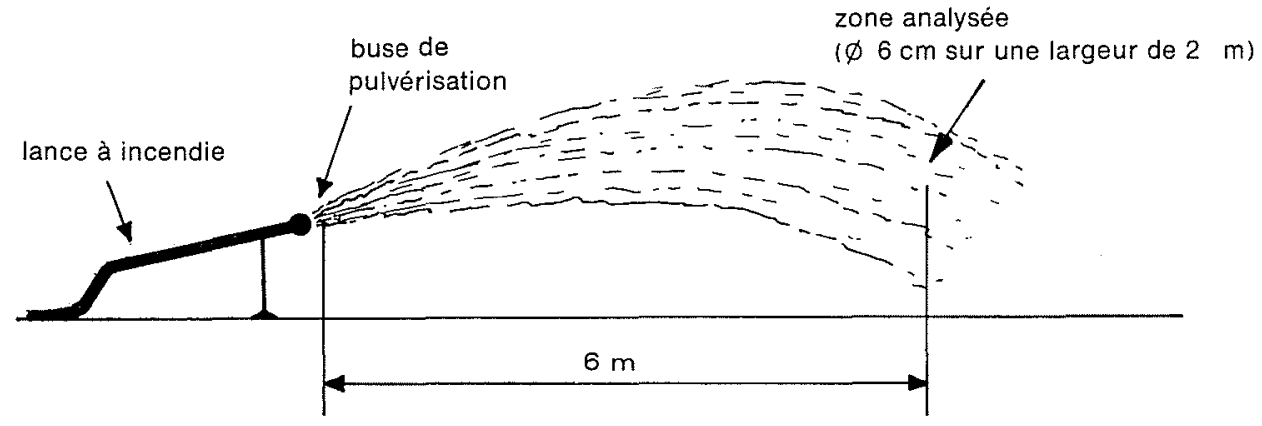


PLANCHE 2

GRANULOMÉTRIE DES GOUTTES

FORMÉES PAR L'ARRACHEMENT D'UN FILM D'EAU AUU BORD DE FUITE D’UNE ĀUBE SOUS L'ACTION D'UN ÉCOULEMENT D'AIR

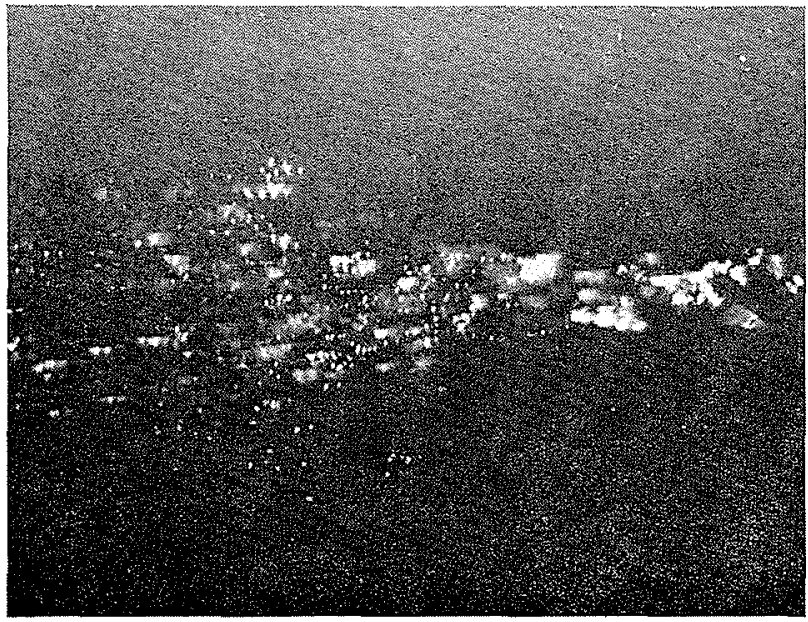

(Image virtuelle)

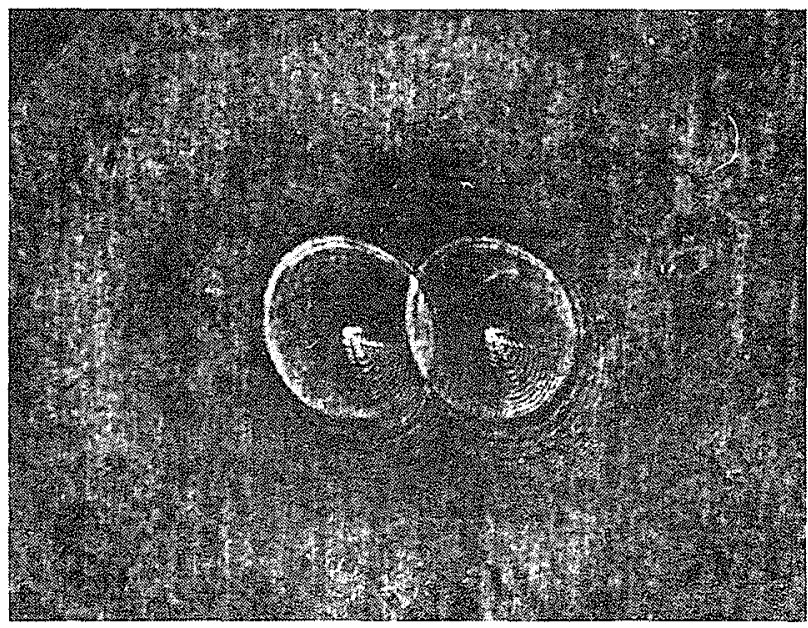

$d=320 \mu$
$N=1$

$g=50$

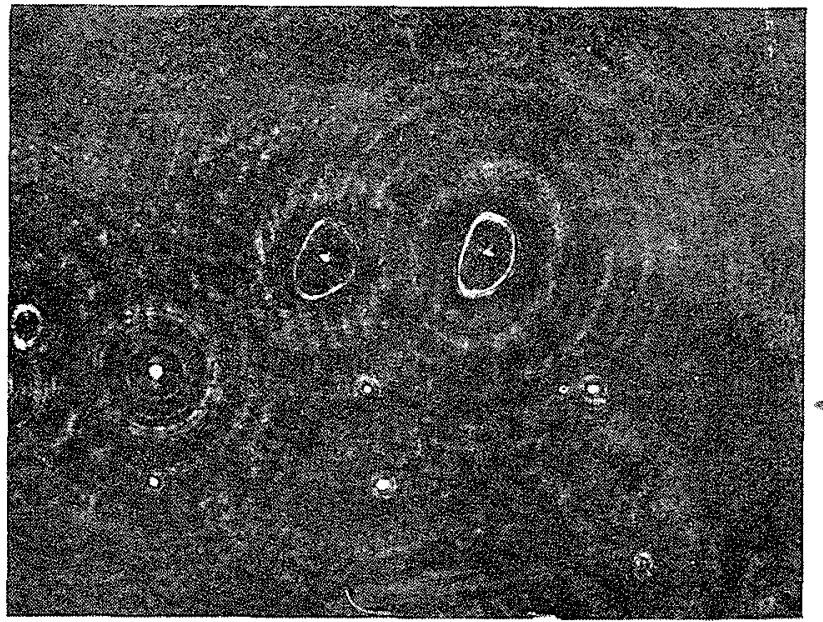

$d=500 \mu$

$N=0,4$

$g=10$

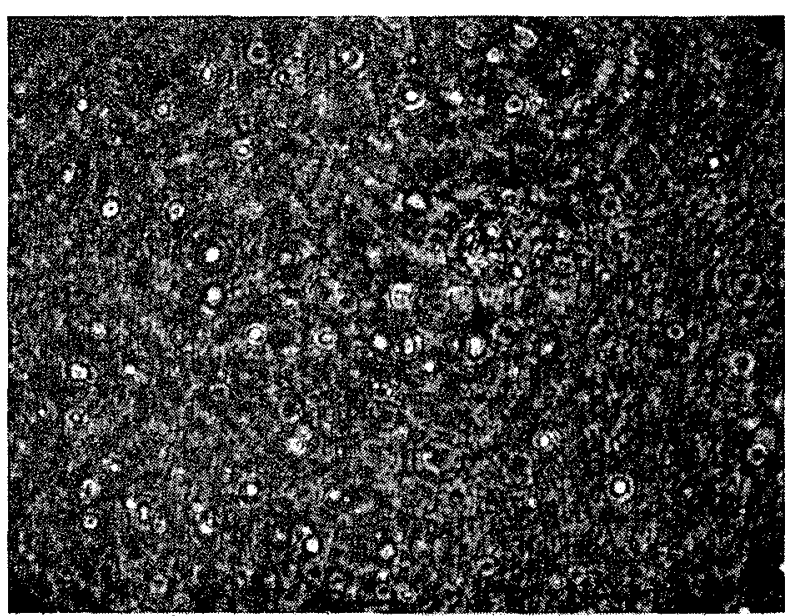

$g=10$

Les hologrammes ci-dessus sont pris en double exposition

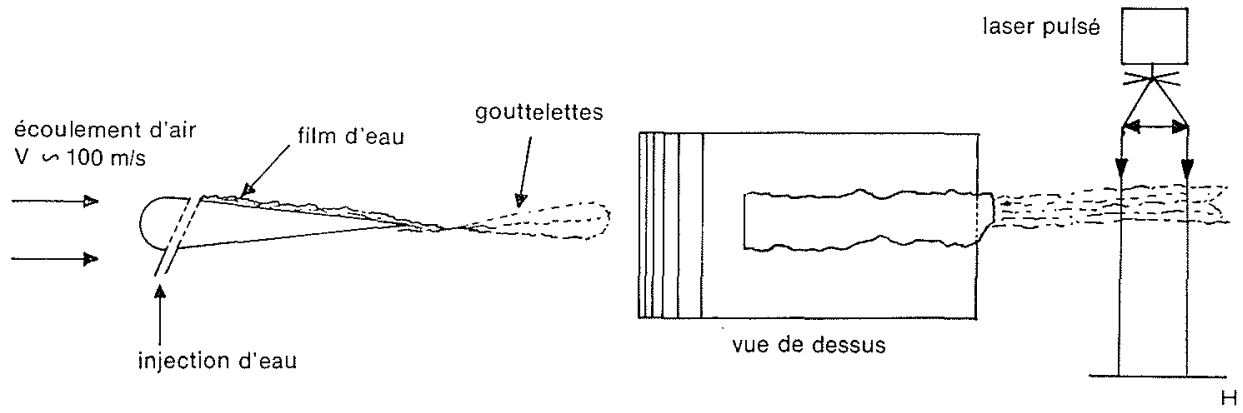




\section{ANALYSE D'UN BROUILLARD DE PULVÉRISATION D'ORIGINE MÉCANIQUE}

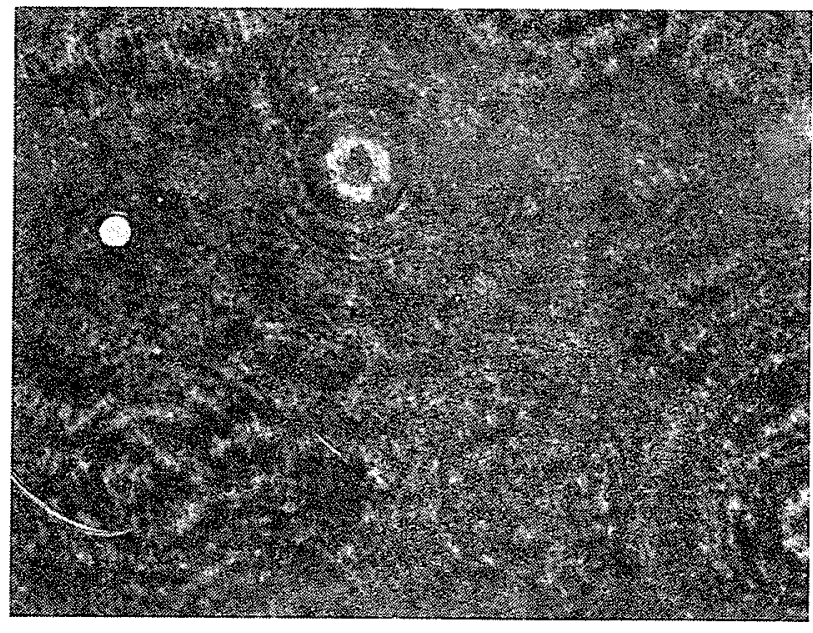
1/ $d \sim 60 \mu$
$N=8$
$g=50$

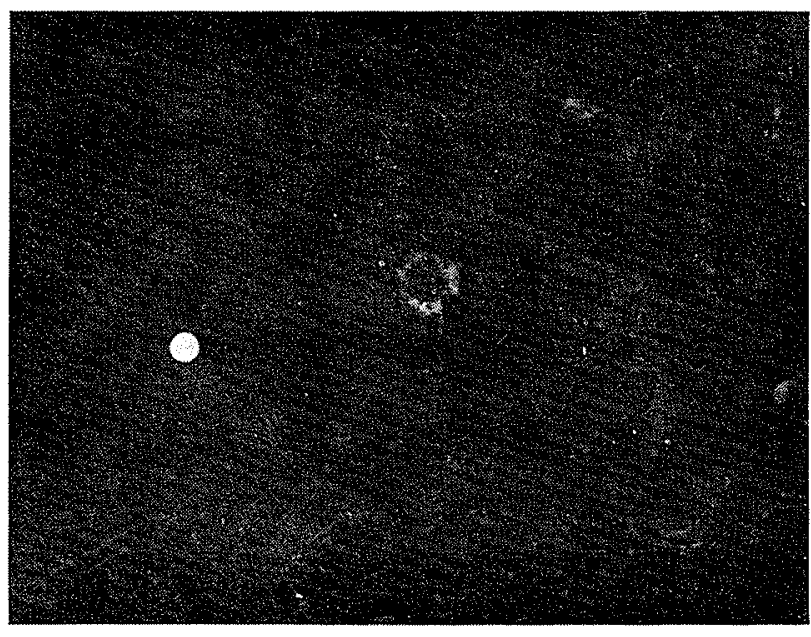

3/ $d \sim 60 \mu$

$N=8$

$g=50$

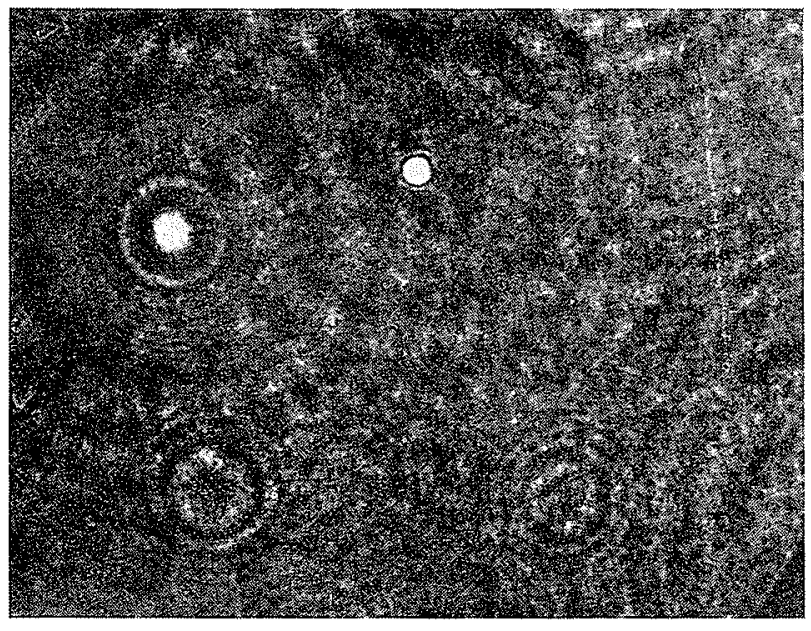

2/

$N=8$

$g=50$

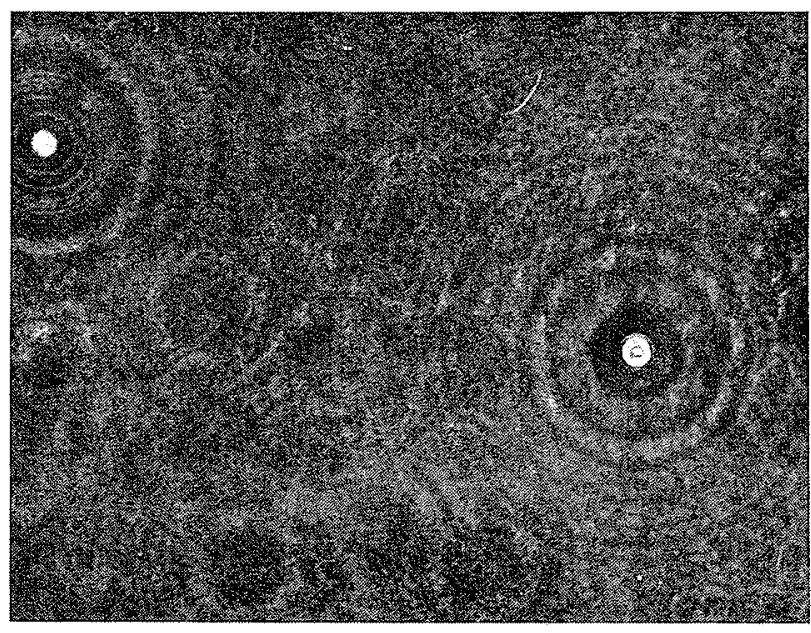

4/ $d \sim 60 \mu \quad N=1,5 \quad g=50$

- Les clichés 1 et 2 représentent des mises au point sur deux gouttes voisines, l'écart entre les deux plans est de $6 \mathrm{~mm}$.

- Les clichés 1 et 3 diffèrent uniquement par la durée du temps de pose. 
PLANCHE 4

\section{ANALYSE D'UN BROUILLARD DE PULVÉRISATION \\ D'ORIGINE PNEUMATIQUE}

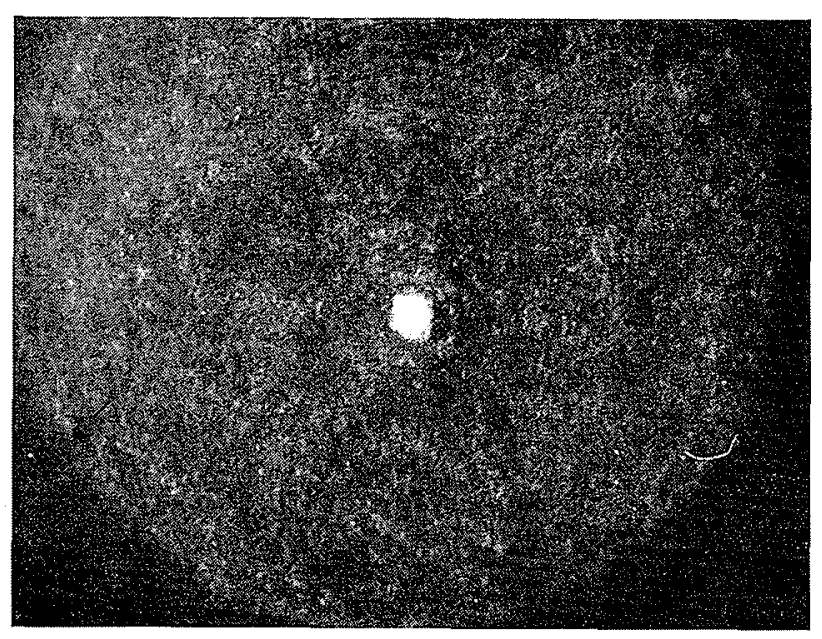

$d=10 \mu \quad g=500$

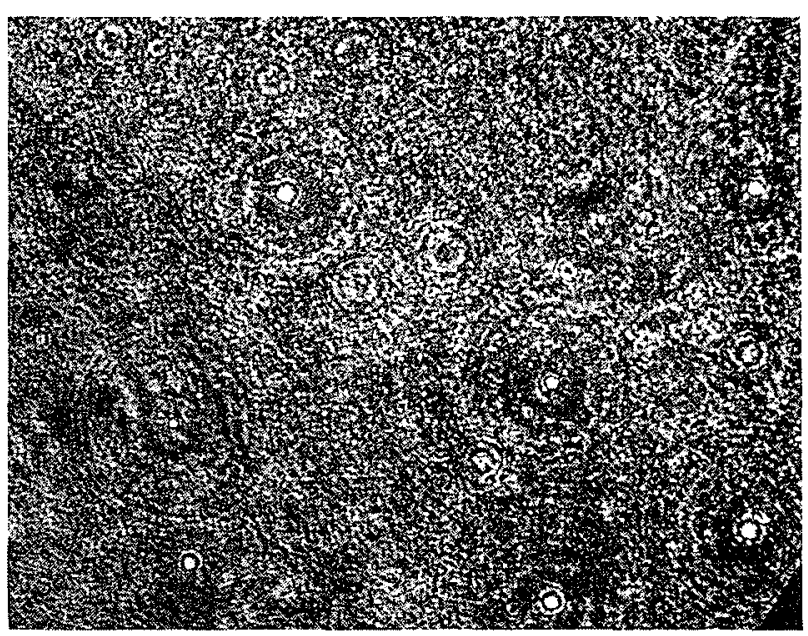

$d=10 \mu$

$N=20$

$g=50$

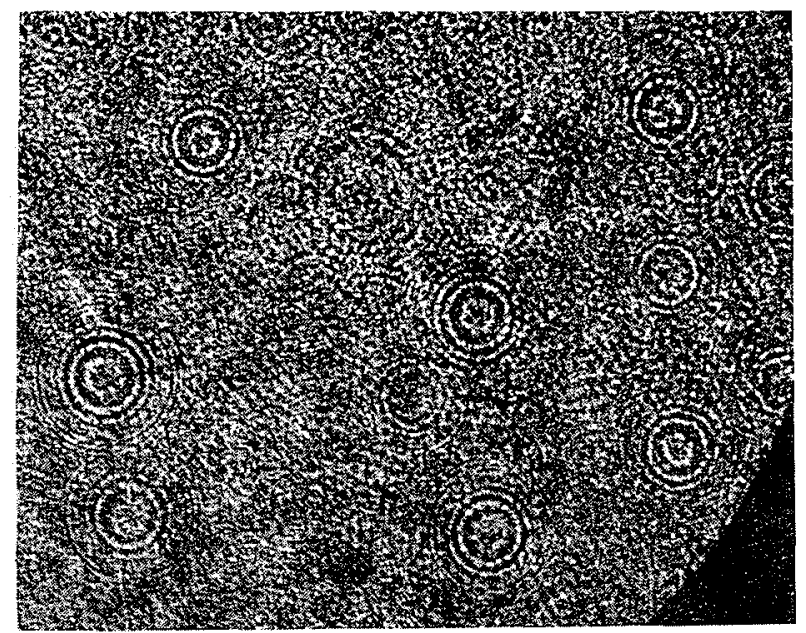

Anneaux de diffraction enregistrés sur

la plaque sensible 


\section{PLANCHE 5}

MONTAGE AVEC REPORT D'OBJETS

(Etude sur micromètre)

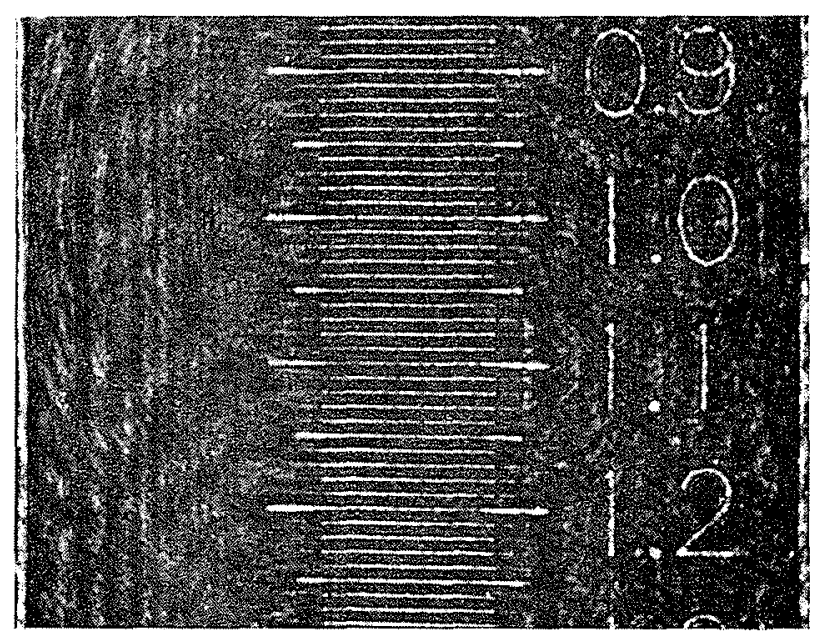

Micromètre

$g=130$

L'épaisseur de graduation

est de $3 \mu$

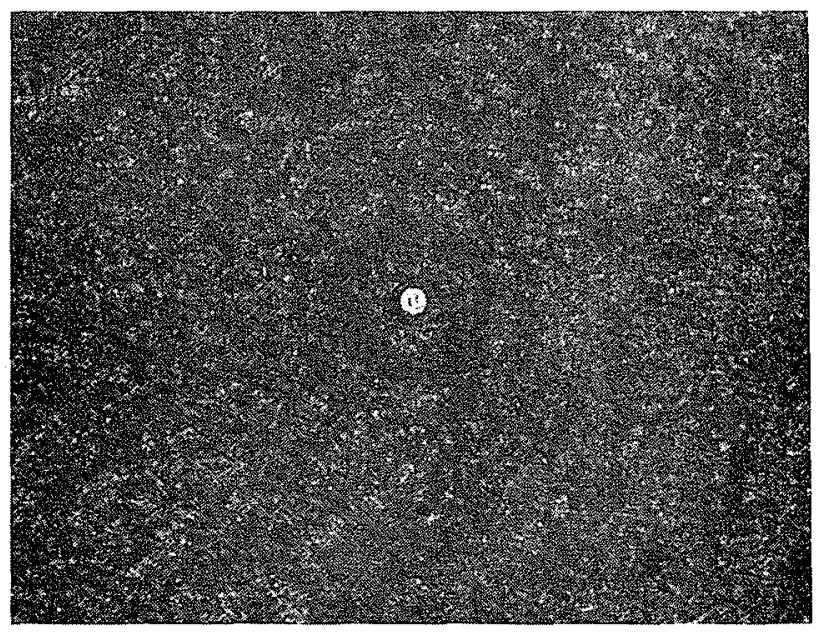

$$
\begin{gathered}
\text { Goutte } \\
10 \mu \\
g=130
\end{gathered}
$$


PLANCHE 6

BULLES D'AIR DANS L'EAU

(Etude de la cavilation)

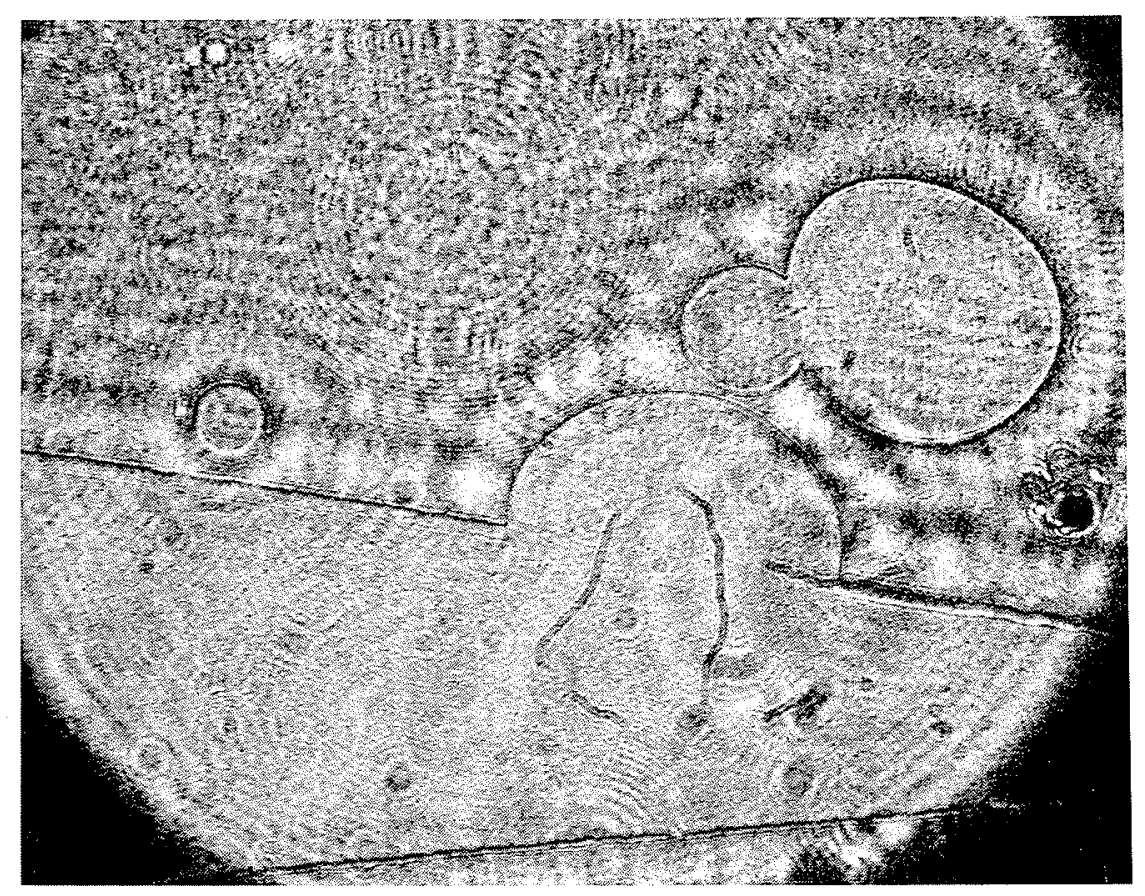

Bulles de l'ordre du millimètre

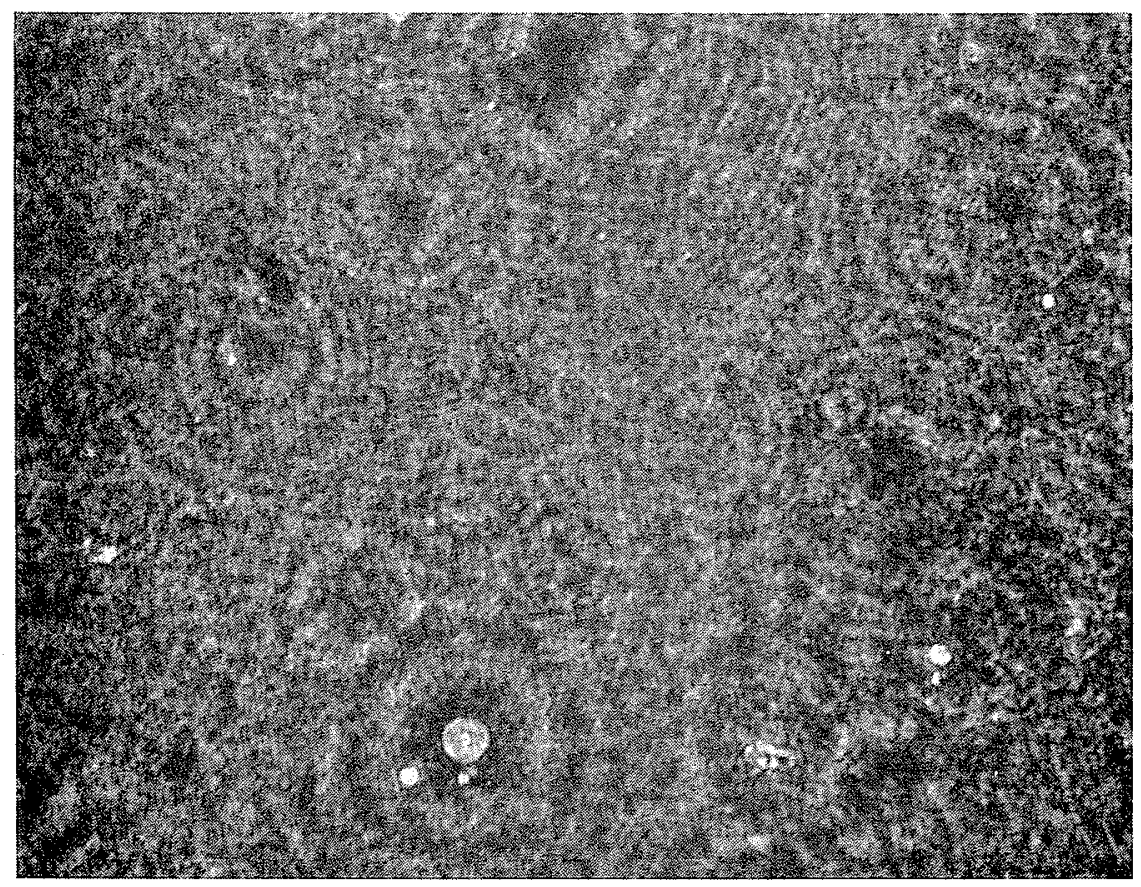

Bulles de 20 à $500 \mu$ 